\title{
UK Food Standards Agency Optimal Nutrition Status Workshop: environmental factors that affect bone health throughout life
}

\author{
Lynn Burns ${ }^{1}$, Margaret Ashwell ${ }^{2}$, Jacqueline Berry ${ }^{3}$, Caroline Bolton-Smith ${ }^{4}$, Aedin Cassidy ${ }^{5}$, \\ Matthew Dunnigan ${ }^{6}$, Kay Tee Khaw ${ }^{7}$, Helen Macdonald ${ }^{8}$, Susan New ${ }^{9}$, Ann Prentice ${ }^{4}$, \\ Jonathan Powell ${ }^{10}$, Jonathan Reeve ${ }^{7}$, Simon Robins ${ }^{11}$ and Birgit Teucher ${ }^{12}$ \\ ${ }^{1}$ Food Standards Agency, Aviation House 125 Kingsway, London WC2 6NH, UK \\ ${ }^{2}$ Ashwell Associates, Ashwell Street, Ashwell, Hertfordshire SG7 5PZ, UK \\ ${ }^{3}$ Vitamin D Research Group, The Medical School, Manchester Royal Infirmary, Oxford Road, Manchester M13 9WL, UK \\ ${ }^{4}$ MRC Human Nutrition Research (HNR), Elsie Widdowson Laboratory, Fulbourn Road, Cambridge CB1 9NL, UK \\ ${ }^{5}$ Unilever, Colworth House, Sharnbrook, Bedford MK44 1LQ, UK \\ ${ }^{6}$ Department of Human Nutrition, Glasgow Royal Infirmary, Glasgow G31 2ER, UK \\ ${ }^{7}$ University of Cambridge, Clinical Gerontology Unit, Addenbrooke's Hospital, Cambridge CB2 2QQ, UK \\ ${ }^{8}$ University of Aberdeen, Medicine and Therapeutics, Osteoporosis Research Unit, \\ Woolmanhill Hospital, Aberdeen AB25 1LD, Scotland, UK \\ ${ }^{9}$ School of Biomedical and Life Sciences, University of Surrey, Guildford, Surrey GU2 7XH, UK \\ ${ }^{10}$ Department of Nutrition and Dietetics, Kings College London, Franklin Wilkins Building, \\ 150 Stamford Street, London SE1 9NN, UK \\ ${ }^{11}$ The Rowett Research Institute, Greenburn Road, Bucksburn, Aberdeen AB21 9SB, Scotland, UK \\ ${ }^{12}$ Institute of Food Research, Norwich Research Park, Colney, Norwich NR4 7UA, UK
}

(Received 17 December 2002 - Revised 17 February 2003 - Accepted 17 February 2003)

\begin{abstract}
The UK Food Standards Agency (FSA) convened a group of expert scientists to discuss and review UK FSA- and Department of Health-funded research on diet and bone health. This research focused on the lifestyle factors that are amenable to change and may significantly affect bone health and the risk of osteoporotic fracture. The potential benefits of fruits and vegetables, meat, $\mathrm{Ca}$, vitamins $\mathrm{D}$ and $\mathrm{K}$ and phyto-oestrogens were presented and discussed. Other lifestyle factors were also discussed, particularly the effect of physical activity and possible gene-nutrient interactions affecting bone health.
\end{abstract}

Bone: Osteoporosis: Fracture: Calcium: Vitamin D: Vitamin K: Phyto-oestrogens: Salt: Silicon: Physical activity

The UK Food Standards Agency (FSA) held a workshop in June 2002 to bring together UK Government-funded researchers investigating the effects of diet and other lifestyle factors on bone health. The aim of the meeting was to share the results of these projects and to determine the progress made since the publication of the UK Committee of Medical Aspects of Food and Nutrition Policy report on nutrition and bone health (Department of Health, 1998).

\section{Background}

Osteoporosis is characterised by low bone mass and microarchitectural deterioration of bone tissue, leading to enhanced bone fragility and a consequent increased risk of bone fracture (World Health Organization, 1994). The most common fractures are of the wrist, hip and spine. It is a painful condition that results in significant morbidity, reduced quality of life and increased mortality.

Currently in the UK, it is expected that one in three women and one in twelve men $>50$ years old will suffer an osteoporotic fracture (Barlow et al. 2001; Department of Health, 2001). The financial costs to the UK National Health Service are enormous and set to rise as the population ages. The estimated annual cost of osteoporosis to the UK National Health Service and Social Services, as stated in the National Framework for Older People 
(Department of Health, 2001), is $£ 1.7$ billion. In addition, the social and personal costs to those affected by osteoporosis are considerable. Fractures, particularly of the hip, can result in permanent disability and loss of independence. Vertebral fractures can occur spontaneously during everyday activities, causing pain and deformity.

Endogenous factors such as genetics, gender, hormonal status and ethnicity contribute greatly to the risk of osteoporosis. With the exception of hormonal factors that can be modulated with hormone treatments, e.g. hormone replacement therapy, these factors cannot be altered.

There is, however, great interest in environmental factors such as nutrition and physical activity, which may have a role in the aetiology of this metabolic bone disease. These factors are amenable to change and are key components of a lifestyle strategy to achieve and maintain optimum bone health at a population level. The nutritional factors were the main focus of the workshop.

\section{Fruits and vegetables}

There is growing epidemiological evidence that fruits and vegetables are beneficial for bone health (Tucker et al. 1999; Sellmeyer et al. 2001). What remains to be determined are the mechanisms for such an effect. A possible mechanism is based on the role that the skeleton plays in acid-base homeostasis (New, 2002). Alternatively, the link between fruit and vegetables and bone may be due to some other 'dietary' component rather than an alkaliaccess effect.

Drs Susan New (Guildford, UK) and Helen Macdonald (Aberdeen, UK) reported the findings of two projects within the Aberdeen Prospective Osteoporosis Screening Study (APOSS). APOSS began in 1990 with the random selection of $>5000$ women aged 45-54 years. Between 1990 and 1993, these women had bone mineral density (BMD) measurements taken at the hip (femoral neck) and spine (lumbar spine). In 1993 a subset of 1064 healthy, mainly pre-menopausal women completed a food-frequency questionnaire and a physical-activity questionnaire. Nutrients associated cross-sectionally with increased axial and peripheral $\mathrm{BMD}$ included $\mathrm{K}, \mathrm{Mg}$, vitamin $\mathrm{C}$, fibre (NSP) and Zn.

A follow-up study, funded by the UK Department of Health and the Medical Research Council between 1997 and 2000, involved a second bone scan and food-frequency questionnaire analysis of 907 women. This showed that in pre-menopausal women, higher intakes of $\mathrm{K}$ and vitamin $\mathrm{C}$ were associated with significantly less bone loss at the femoral neck. Subsequent FSA (N05026) funding allowed 3239 out of the total 5000 women to complete the food-frequency questionnaire. $\mathrm{A}$ high $\mathrm{Mg}$ intake was associated with increased hip BMD and, for pre-menopausal women, fruit and vegetable intake was associated with greater BMD at both the lumbar spine and the hip. Similar findings were observed in subjects who had previously used hormone-replacement therapy.

Dr Jonathan Reeve (Cambridge, UK) presented results from an FSA-funded analysis of the European Prospective Osteoporosis Study cohort from the large EPIC-Norfolk prospective study. In women, low intake of vitamin $\mathrm{C}$ was associated with a faster rate of bone loss, which was not seen in men. Vitamin $\mathrm{C}$ is required for hydroxylation of collagen and this may be why vitamin $\mathrm{C}$ is important for bone formation. Vitamin $\mathrm{C}$ intake and status is often used as an indicator of fruit and vegetable consumption and these associations may be indicative of a role for fruits and vegetables that is not due to their vitamin $\mathrm{C}$ content per se. Nutrients such as $\mathrm{Mg}$ and $\mathrm{K}$ are abundant in fruits and vegetables; associations between their intakes and increased BMD have also been observed in other studies (Tucker et al. 1999).

It is possible that the alkaline nature of fruits and vegetables promotes bone health. Several amino acids present in meat and cheese produce $\mathrm{H}^{+}$ions in their metabolism and cause a lowering of blood $\mathrm{pH}$. These $\mathrm{H}^{+}$ions are generally removed by the kidneys, but if the $\mathrm{pH}$ continues to fall, the skeleton acts as a buffer by releasing carbonates and phosphates. Alkaline salts produced during the metabolism of fruits and vegetables can also neutralise the $\mathrm{H}^{+}$ ions. So consumption of fruits and vegetables that produce alkaline salts, such as carbonates and citrates, reduces the need for the bones to provide part of the buffer system. With increasing age, the kidneys become less efficient at removing the $\mathrm{H}^{+}$ions from the blood and hence the importance of consuming foods that help to counteract the acidproducing foods becomes even greater.

One way to estimate the acid produced by the diet is to calculate the net endogenous acid production, which is the dietary protein : dietary K ratio (Frassetto et al. 1998). Macdonald and New presented results for post-menopausal women in the FSA subset within the longitudinal APOSS cohort showing that low intakes of vegetables and high estimates of net endogenous acid production were associated with increased levels of bone resorption markers. The Aberdeen group is starting a new project using APOSS subjects stratified according to genotypes possibly influencing bone health, to explore the beneficial mechanism of fruits and vegetables in greater detail.

\section{Phyto-oestrogens}

The similarity in structure of phyto-oestrogens (e.g. isoflavones) to human oestrogens results in them having a weak oestrogenic effect and their potential protective effects on bone health have received considerable attention (Setchell \& Cassidy, 1999). Dr Simon Robins (Aberdeen, UK) explained that bone metabolism represents a complex series of events mediated by bone-forming osteoblastic cells and osteoclastic cells involved in bone resorption. These cells, together with osteocytes, orchestrate the bone remodelling cycle that is so susceptible to perturbations by hormonal and other factors.

All bone cells contain oestrogen receptors, so a possible mechanism for isoflavone action is a direct interaction with these receptors. There are two main oestrogen receptors, $\alpha$ and $\beta$. Different phyto-oestrogens bind to these receptors to varying degrees; however, their binding affinities are many orders of magnitude less than that of oestradiol. Stronger interactions occur with the oestrogen receptor- $\beta$, but the outcome of such interactions is difficult to predict. The recent observation that transgenic mice lacking 
oestrogen receptor- $\beta$ have increased bone mass compared with their normal counterparts provides additional evidence for the action of oestrogen receptor- $\beta$ as a negative regulator in bone, thus raising additional questions about the mechanisms of action of phyto-oestrogens.

Dr Sheila Bingham's (Cambridge, UK) FSA-funded double-blind placebo-controlled trial (presented by Dr Robins) involved pre-, peri- and post-menopausal women (aged 49-65 years) who were not on hormone replacement therapy. The test group were given $40 \mathrm{mg}$ isoflavones/d (a combination of two isoflavones, genistein and daidzein), for 1 year (equivalent to a daily meal of soyabean). Compliance was good and was tested by measuring isoflavone metabolites in the urine. In the lumbar spine, significantly less bone mineral content and BMD was lost, especially in pre-menopausal women in the test group. There was a significantly greater increase in the bone formation marker amino-terminal propeptide of type I procollagen (PINP) amongst women taking the isoflavone supplement compared with those taking the placebo, especially in post-menopausal women. There were no significant treatment effects on hip bone mineral content or density or on other markers of bone turnover, pyridinoline and deoxypyridinoline. These results suggest a potentially protective effect of phyto-oestrogens on the maintenance of bone density and in bone formation in women at, or around, the menopause. However, there were contrasting effects between the markers of bone formation and bone density, which were dependent on menopausal status.

Several other mechanisms of action have been postulated based on the known effects of oestradiol on bone. These include actions on cytokines and growth factors and their associated regulatory molecules, as well as enzymes involved in signal transduction, cell proliferation and apoptosis. Certain isoflavones have also been shown to inhibit the action of aromatase, thus potentially limiting the synthesis of endogenous oestrogens.

From a large number of animal studies, mainly in rats, there is generally good evidence that dietary isoflavones both decrease bone resorption and increase bone formation rates. Most of these studies, however, involve much higher doses of the plant compounds than those used in human trials.

Several other intervention trials with phyto-oestrogens in healthy volunteers have been reported using BMD and biochemical markers of bone metabolism as the primary outcome measures. The results so far are inconclusive, mainly because most of the studies are relatively short-term in relation to the rate of change in skeletal metabolism. It is hoped that the results from ongoing, longer-term studies will provide definitive evidence on which to judge whether dietary supplementation with isoflavones is really beneficial to bone health.

Dr Aedin Cassidy (Unilever, UK) described an ongoing European Union project, PHYTOS, which aims to determine the biological effects of isoflavone intervention on bone density and bone metabolism in post-menopausal European women. Women ( $n$ 300) in three European Union countries (France, Italy and the Netherlands) will be given isoflavone-enriched foods or placebo for 12 months and will be monitored at several time-points.

\section{Vitamin K}

Vitamin K, found as phylloquinone in the diet, is increasingly being considered as an important nutrient for bone health (Shearer, 1997). Vitamin K mediates the $\gamma$-carboxylation of glutamyl residues on several bone proteins, in particular, osteocalcin. This protein, which is formed by the osteoblasts, is often used as a marker of bone formation. Osteocalcin, when $\gamma$-carboxylated, binds $\mathrm{Ca}$ ions in the hydroxyapatite molecule and may regulate structural aspects of bone formation and $\mathrm{Ca}$ balance (Weber, 2001).

A small amount of osteocalcin circulates in the serum and levels of undercarboxylated osteocalcin have been found to be a better measure of vitamin $\mathrm{K}$ status than the traditional blood coagulation tests. High levels of undercarboxylated osteocalcin have been associated with lower BMD. Dr Jonathan Reeve reported a significant positive association between under-carboxylated osteocalcin levels and bone loss after 18 months in his prospective study in Norfolk (UK) women. It will be interesting to see if this association still stands when greater bone loss has occurred.

It has been suggested that there is a role for vitamin D in the functional nature of osteocalcin. Dr Caroline BoltonSmith (Cambridge, UK) and Dr Martin Shearer (London, UK) investigated the possible synergy between vitamins $\mathrm{D}$ and $\mathrm{K}$ in a group of Dundee (UK) women (aged 6085 years) in a 2-year randomised placebo-controlled parallel-group study. The four intervention groups were: placebo, $200 \mu \mathrm{g}$ phylloquinone/d, $10 \mu \mathrm{g}$ vitamin $\mathrm{D}$ and $1 \mathrm{~g}$ $\mathrm{Ca} / \mathrm{d}, 200 \mu \mathrm{g}$ phylloquinone, $10 \mu \mathrm{g}$ vitamin $\mathrm{D}$ and $1 \mathrm{~g} \mathrm{Ca} /$ $\mathrm{d}$. The rationale for the choice of supplementation was as follows: $10 \mu \mathrm{g}$ vitamin $\mathrm{D}$ is the recommended amount for supplementing the elderly and is easily obtainable from a portion of oil-rich fish; $200 \mu \mathrm{g}$ vitamin $\mathrm{K}$ is easily obtained from $100 \mathrm{~g}$ portion of leafy green vegetables; $1 \mathrm{~g} \mathrm{Ca}$ was to ensure adequacy.

BMD was measured by dual-energy x-ray absorptiometry at the hip (three sites) and at the wrist (three sites). Dietary and other lifestyle information, including activity and sunlight exposure, were also collected every 6 months. There was evidence of sub-optimal levels of vitamin $\mathrm{D}$ and $\mathrm{K}$ in the study groups, which were improved with supplementation. In the group provided with vitamins $\mathrm{D}$ and $\mathrm{K}$, there was a significant conservation of BMD at one of the wrist sites (ultra-distal radius). No significant changes were seen at any other sites.

Many workshop participants had noted that the rate of bone mineral loss of subjects in the reported trials was much lower than anticipated. There was agreement that this needs to be taken into account for future studies to ensure statistical power.

\section{Meat and vitamin D}

Vitamin D is known to be extremely important for bone health (Mawer \& Davies, 2001). The main source of vitamin $\mathrm{D}$ is that made in the skin following exposure to sunlight. The availability of vitamin D from this source depends on season and latitude as well as the extent of skin exposure, its pigmentation and thickness. Dietary 
sources are limited, the richest sources being oil-rich fish, butter and other fat spreads, eggs and meat. The paradox that most vitamin $\mathrm{D}$ is derived from synthesis in the skin, while severe rickets and osteomalacia are invariably associated with varying degrees of vegetarianism remains unanswered, given the low dietary vitamin D content of the largely unfortified British diet.

Dr Matthew Dunnigan (Glasgow, UK) and Dr Jacqueline Berry (Manchester, UK) presented the results of the study headed by Professor Barbara Mawer (Manchester, UK), which looked at the importance of meat consumption in regulating vitamin $\mathrm{D}$ metabolism and promoting bone health. Study participants were vegetarian and omnivore women, both Asian and Caucasian. A vegetarian diet and Asian ethnicity were both associated with lower vitamin D status and BMD. Significantly decreasing trends were found in serum 25-hydroxycholecalciferol levels and trabecular and cortical BMD as follows: white omnivores $>$ white vegetarians $>$ Asian omnivores $>$ Asian vegetarians. The absence of meat was the only dietary factor related to the lower BMD of vegetarians. There was no apparent relationship between the quantity of meat eaten and the BMD or vitamin D status.

Women supplemented with either a daily oral dose of $400 \mathrm{IU}(10 \mu \mathrm{g})$ ergocalciferol for four weeks or a single large oral dose of $50000 \mathrm{IU}(125 \mu \mathrm{g})$ ergocalciferol did not show any quantitative differences in the metabolism of vitamin D related to habitual diet or ethnicity (as determined by serum 25-hydroxycholecalciferol concentration and measured as area under the response curve). Thus, the poorer vitamin D status and bone health in vegetarian women did not appear to be due to impaired hepatic or renal hydroxylation of administered ergocalciferol.

Contrary to current thinking, the recommended intake of vitamin D (a daily oral dose of $400 \mathrm{IU}(10 \mu \mathrm{g})$ ergocalciferol) for 1 month did not raise the serum concentration of 25-hydroxycholecalciferol to values that indicated adequate vitamin $D$ status $(>15 \mathrm{ng} / \mathrm{ml})$.

The current assessment of the vitamin D content of meat (about $7 \mu \mathrm{g}(28 \mathrm{IU}) / \mathrm{kg}$ ) could not explain its protective role. The researchers suggested that meat could be contributing substantially greater amounts of bioavailable vitamin D than current estimates.

\section{Calcium}

One potential strategy suggested for reducing the incidence of osteoporosis in older people is to optimise peak bone mass in young adults before net bone loss begins. Current public health messages to young people include advice to eat a Ca-rich diet and to take regular exercise. Surprisingly, the scientific evidence underpinning this advice is not overwhelming. (Department of Health, 1998).

Dr Ann Prentice (Cambridge, UK) presented results from the Cambridge Bone Studies, which were designed to test the impact of $\mathrm{Ca}$ supplementation and exercise on the bone mineral status of 16-18-year-old boys and girls. Two separate studies with similar protocols were conducted and both included follow-up measurements 1 year after the intervention phase.
At enrolment, the recruits in the boys' intervention study (mean age 16.8 (SD 0.5) years) were classified into a high or low customary activity group based on their regular participation in sport. After stratification, the boys were randomly assigned, double blind, to receive either a $\mathrm{Ca}$ supplement (1000 mg Ca as calcium carbonate/d) or a matching placebo for 12 months. The participants in the low-activity group were then randomly allocated to one of two exercise groups, and stratified by supplement group. The exercise group, but not the other group, was invited and encouraged to attend three exercise classes per week. Of the boys, 143 completed the study and were included in the final dataset.

The girls' intervention study followed a similar recruitment and allocation protocol. In general, the participation of girls in sport was low and classification into high and low customary activity was not necessary. Of the girls, 131 completed the study and were included into the final dataset. The $\mathrm{Ca}$ intervention period for the girls was 15.5 months.

The results clearly demonstrated that increasing the $\mathrm{Ca}$ intake of boys and girls aged 16-18 years above their customary intake produces an increase in bone mineral content, especially in the axial skeleton (spine and hip). If maintained into later life, such increases would be expected to decrease the risk of osteoporosis by $10-40 \%$. Evidence was also obtained that participation in regular weight-bearing exercise in adolescence may also be effective in increasing bone mineral content. The impact of $\mathrm{Ca}$ intervention on skeletal growth differed between girls and boys, suggesting that different mechanisms may underlie these effects. Ongoing work is investigating these mechanisms and on the long-term effects of these interventions.

This study demonstrated the difficulties in persuading this age group to participate in physical activity even under intervention conditions and provide powerful messages for policy makers trying to encourage exercise in this age group.

The effect of $\mathrm{Ca}$ on minimising bone loss in older women was also investigated in the APOSS cohort. Within the cohort, the effect of $\mathrm{Ca}$ at the femoral neck may be confounded by its association with the vitamin D-receptor gene. Women with the homozygous recessive bb genotype seemed to show a greater beneficial effect from increased $\mathrm{Ca}$ intake than those with the BB genotype, but only at the lowest quartile of $\mathrm{Ca}$ intake. The effect was no longer significant after adjustment for body size.

\section{Other dietary factors}

Silicon ( $\mathrm{Si}$ ) is present in the diet and tissues of man at significant levels. There is some evidence to suggest a role for $\mathrm{Si}$ in bone health, at least partially through regulation of osteoblast function and osteocalcin synthesis. Dr Jonathan Powell (London, UK) explained the aims of FSA-funded research to investigate this hypothesis using secondary analysis of the women who took part in the vitamin $\mathrm{K}$ and D study in Dundee (Scotland, UK).

It is well documented, but only from short-term studies, that a high-Na diet increases urinary $\mathrm{Ca}$ excretion (Massey 
\&Whiting, 1996). If not compensated for, a prolonged intake may increase bone resorption and subsequently increase bone loss. Dr Birgit Teucher (Norwich, UK) outlined her dietary intervention study investigating the effect of high$\mathrm{Na}$ intakes on $\mathrm{Ca}$ and bone metabolism in twenty post-menopausal women. This project is part of a large European Union Framework 5-funded programme on optimal nutrition towards osteoporosis prevention (Osteodiet).

\section{Non-dietary factors}

The risk of fracture is affected by many external factors in addition to diet. Professor Kay Tee Khaw (Cambridge, UK) presented work carried out within the EPIC-Norfolk cohort using bone ultrasound attenuation at the heel, measured in 15668 men and women. Fractures requiring hospital admission were identified within the cohort and early analyses indicate that bone ultrasound attenuation can be a powerful predictor of total and hip fractures in cohorts of this size. Those in the lowest $10 \%$ of the bone ultrasound attenuation distribution had an approximately fourfold increase in fracture risk.

High-impact physical activity was independently associated with bone ultrasound attenuation in both men and women. In addition, bone ultrasound attenuation was positively associated with climbing stairs in women and negatively associated with television watching.

There is also an increased fracture risk in smokers; this effect which is strongest in women, with a twofold risk of fracture for current smokers compared with current nonsmokers.

Reeve's analysis of the EPIC-Norfolk cohort also showed that commonly practised forms of physical activity appeared to protect against bone loss. Weight maintenance was also shown to be extremely important. In both men and women, weight gain protected against bone loss and weight loss promoted bone loss. Measures to ensure general nutrition to guard against weight loss in the non-overweight elderly could be of great value in protecting against fracture.

Although genetic make-up cannot be altered, there may be considerable benefits of changes in diet for those with particular genotypes since there is increasing evidence for the possibility of gene-nutrient interactions. APOSS found some evidence for an interaction between $\mathrm{Ca}$ intake and the gene for the vitamin $\mathrm{D}$ receptor. More research is required in the area of gene-nutrient interactions; population strategies could have a far greater effect on fracture risk than currently estimated.

\section{Research suggestions}

The research findings presented here support the importance of the UK Government's healthy lifestyle guidelines for bone health. These guidelines recommend a balanced diet containing a variety of foods including plenty of fruits and vegetables, as well as participation in weightbearing physical activity and the avoidance of smoking. This research highlights the importance of these factors for maintaining a healthy skeleton. However, further work is required to ascertain the extent of these benefits in order to provide specific nutritional advice for bone health to the general public. Several suggestions were made for such research as follows.

Evidence is needed on:

(1) the effect of lifestyle factors on the accretion of bone mass in young adults and children and the interplay of these factors in utero;

(2) the relationship between peak bone mass and fracture risk in later life;

(3) the factors affecting bone health of men (different approaches and methods may be needed);

(4) the value of alternative measures to BMD in predicting fracture, e.g. hip strength and bone ultrasound attenuation;

(5) the effects of individual nutrients, both in isolation and in combination, including protein and micronutrients, e.g. $\mathrm{Cu}$ and $\mathrm{Zn}$, and of other dietary factors, e.g. the 'meat factor' and factors affecting bioavailability of nutrients.

For vitamin D, more evidence is required to:

(1) determine the most appropriate values for plasma 25hydroxyxholecalciferol to define deficiency, insufficiency and repletion;

(2) determine how much sunlight exposure provides sufficient vitamin D across different age and ethnic groups;

(3) determine its role in muscle function and the possible reduction in fractures by the prevention of falls.

Ideally, research should:

(1) use disease endpoints i.e. fracture incidence, as outcome measures;

(2) use appropriate analytical methods to probe dietary patterns as well as diet quality on bone health (the multi-factorial nature of the interactions inherently requires the development of sophisticated statistical methods);

(3) take into account possible effects of age, gender and key genes that may influence bone health;

(4) be followed by food-based work to develop sound food-based recommendations that can be effectively implemented.

Finally, the participants stressed the importance of the implementation of previous recommendations. The UK Department of Health report The Nutrition of Elderly People (Department of Health, 1992) suggested that vitamin D supplementation should be considered during winter and early spring if adequate exposure to sunlight is not possible. However, very few elderly care-home residents currently receive supplements.

\section{References}

Barlow DH, Roger M Francis \& Andrew Miles (2001) The Effective Management of Osteoporosis. London: Aesculapius Medical Press.

Department of Health (1992) The Nutrition of Elderly People. Committee on Medical Aspects of Food Policy. London: H. M. Stationery Office. 
Department of Health (1998) Nutrition and Bone Health, With Particular Reference to Calcium and Vitamin D. Committee on the Medical Aspects of Food and Nutrition Policy. Working Group on the Nutritional Status of the Population Subgroup on Bone Health. London: The Stationery Office.

Frassetto LA, Todd KM, Morris RC Jr \& Sebastian A (1998) Estimation of net endogenous non-carbonic acid production in humans from diet potassium and protein contents. American Journal of Clinical Nutrition 68, 576-583.

Mawer EB \& Davies M (2001) Vitamin D nutrition and bone disease in adults. Reviews in Endocrine and Metabolic Disorders 2, 153-164.

Department of Health (2001) National Service Framework for Older People. London: The Stationery Office.

New SA (2002) The role of the skeleton in acid-base homeostasis. The 2001 UK Nutrition Society Medal Lecture. Proceedings of the Nutrition Society 61, 151-164.

Massey LK \& Whiting SJ (1996) Dietary salt, urinary calcium and bone loss. Journal of Bone Mineral Research 11, 731-736.

Sellmeyer DH, Stone KL, Sebastion A \& Cummings SR (2001) A high ratio of dietary animal to vegetable protein increases the rate of bone loss and the risk of fracture in postmenopausal women. American Journal of Clinical Nutrition 73, 118-122.

Setchell KD \& Cassidy A (1999) Dietary isoflavones: biological effects and relevance to human health. Journal of Nutrition 129, 758S-767S.

Shearer MJ (1997) The roles for vitamins D and K in bone health and osteoporosis prevention. Proceedings of the Nutrition Society 56, 915-937.

Tucker KL, Hannan MT, Chen H, Cupples LA, Wilson PWF \& Kiel DP (1999) Potassium, magnesium and fruit and vegetable intakes are associated with greater bone mineral density in elderly men and women. American Journal of Clinical Nutrition 69, 727-736.

Weber (2001) Vitamin K and bone health. Nutrition 17, 880-887.

World Health Organization (1994) Assessment of Fracture Risk and its Application to Screening for Postmenopausal Osteoporosis: Report of a World Health Organization Study Group. Technical Report. Series no. 843. Geneva: WHO.

\section{Appendix}

Participants in the workshop were: Dr Frazer Anderson, University of Southampton (UK); Dr Margaret Ashwell, Food Standards Agency Advisor (UK); Dr Jacqueline Berry, University of Manchester (UK); Dr Caroline Bolton Smith, HNR Cambridge (UK); Dr Francesco Branca, National Institute for Research on Food and Nutrition, Rome (Italy); Dr Aedin Cassidy, formerly Unilever plc (UK); Dr Matthew Dunnigan, University of Glasgow (UK); Dr Bronwyn Evans, University of Cardiff (UK); Dr Roger Francis, University of Newcastle (UK); Dr Joyce Hughes, Department of Health Advisor (UK); Professor Kay Tee Khaw, University of Cambridge (UK); Dr Helen Macdonald, University of Aberdeen (UK); Professor Hugh Makin, Food Standards Agency Advisor (UK); Dr Susan New, University of Surrey (UK); Dr Jonathan Powell, University of London (UK); Dr Ann Prentice, HNR Cambridge (UK); Dr Sheela Reddy, Department of Health England (UK); Dr Jonathan Reeve, University of Cambridge (UK); Dr Simon Robins, University of Aberdeen (UK); Professor Andrew J. Rugg-Gunn, University of Newcastle (UK); Dr Birgit Teucher, IFR Norwich (UK); Dr Anita Thomas, Derriford Hospital, Plymouth (UK); Ms Ailsa Welch, University of Cambridge (UK); Lynn Burns, FSA (UK); Dr Lisa Jackson, FSA (UK); Robert Martin, FSA (UK); Dr Alison Tedstone, FSA (UK); Ben Walters, FSA (UK); Anna Whyte, FSA Scotland (UK). 